Check for updates

Cite this: RSC Adv., 2019, 9, 9546

Received 14th January 2019

Accepted 14th March 2019

DOI: $10.1039 / c 9 r a 00317 g$

rsc.li/rsc-advances

\section{Effects of temperature and pore structure on the release of methane in zeolite nanochannels}

\author{
Xu Cheng, (D) ${ }^{a}$ Zhigang Li (D) *a and Ya-Ling He ${ }^{* b}$
}

In this work, we investigate the effects of temperature and pore size on the release of methane in zeolite nanochannels through molecular dynamics (MD) simulations. The methane release percentage at different temperatures and for different zeolite structures is calculated. In all-silica MFI (silicalite-1) zeolite, it is found that the release percentage increases with increasing temperature roughly at a constant rate when the temperature is below $598 \mathrm{~K}$. For higher temperatures, the release percentage reaches about $90 \%$ and remains almost constant. For other structures, the release percentage is greatly affected by the average pore size. The release percentage is determined by the temperature and energy barrier inside the pores. Based on the energy barriers obtained in MD simulations, theoretical predictions of the release percentage are made, which are in good agreement with numerical results.

\section{Introduction}

Shale gas, which is predominantly composed of methane, is an important, eco-friendly energy resource. Whether shale gas can change the energy roadmap or not depends on the production cost. In the past decade, with the advances in various

${ }^{a}$ Department of Mechanical and Aerospace Engineering, The Hong Kong University of Science and Technology, Clear Water Bay, Kowloon, Hong Kong. E-mail: mezli@ust.hk ${ }^{b}$ Key Laboratory of Thermo-Fluid Science and Engineering of MOE, School of Energy and Power Engineering, Xi'an Jiaotong University, Xi'an, Shaanxi 710049, PR China. E-mail: yalinghe@mail.xjtu.edu.cn technologies, such as hydraulic fracturing and horizontal drilling, mass production of shale gas has been greatly promoted. As shale gas is confined in organic-rich, nanoscale shale formations, which are of very low permeability, ${ }^{1}$ the development of shale gas is much more difficult than that of conventional natural gases. Furthermore, the output of a typical shale gas well usually experiences a significant drop after three years. ${ }^{2,3}$ Therefore, it is important to understand the transport mechanisms of shale gas/methane in nanoconfinements and find new ways to enhance the release of methane from nanopores. This may lead to significant changes to the energy consumption landscape.

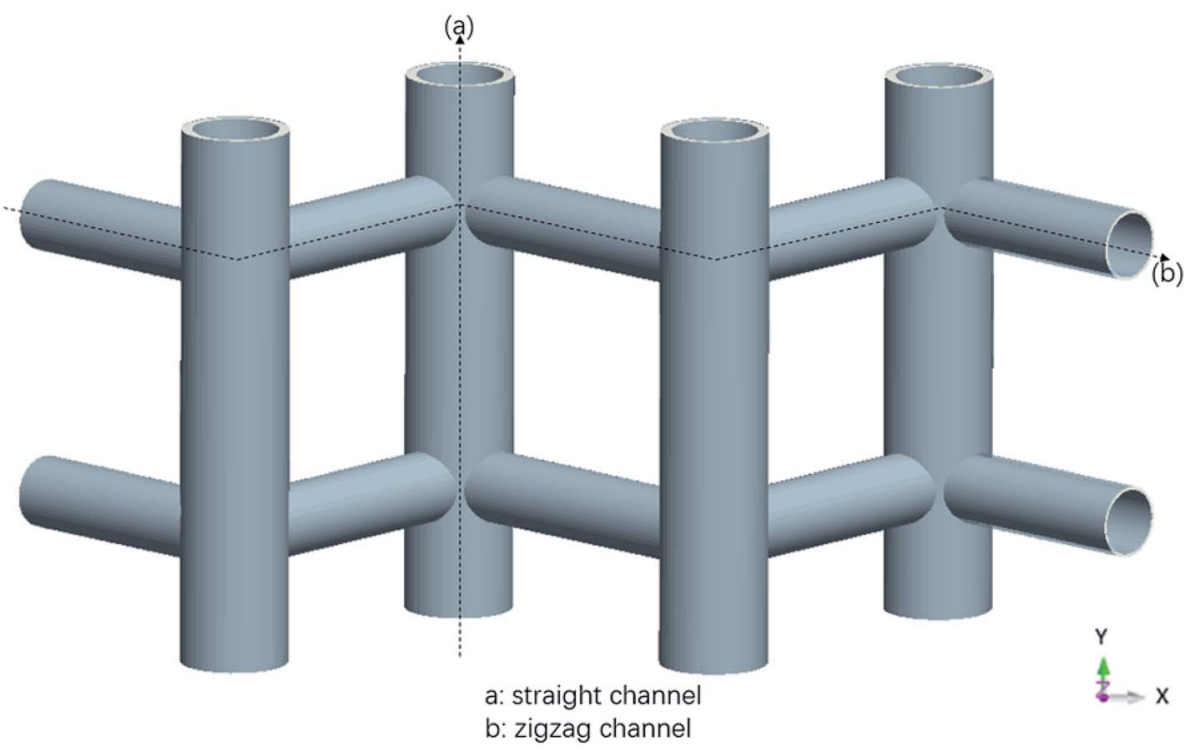

Fig. 1 Schematic of the zeolite pore structure illustrating straight and zigzag channels. 

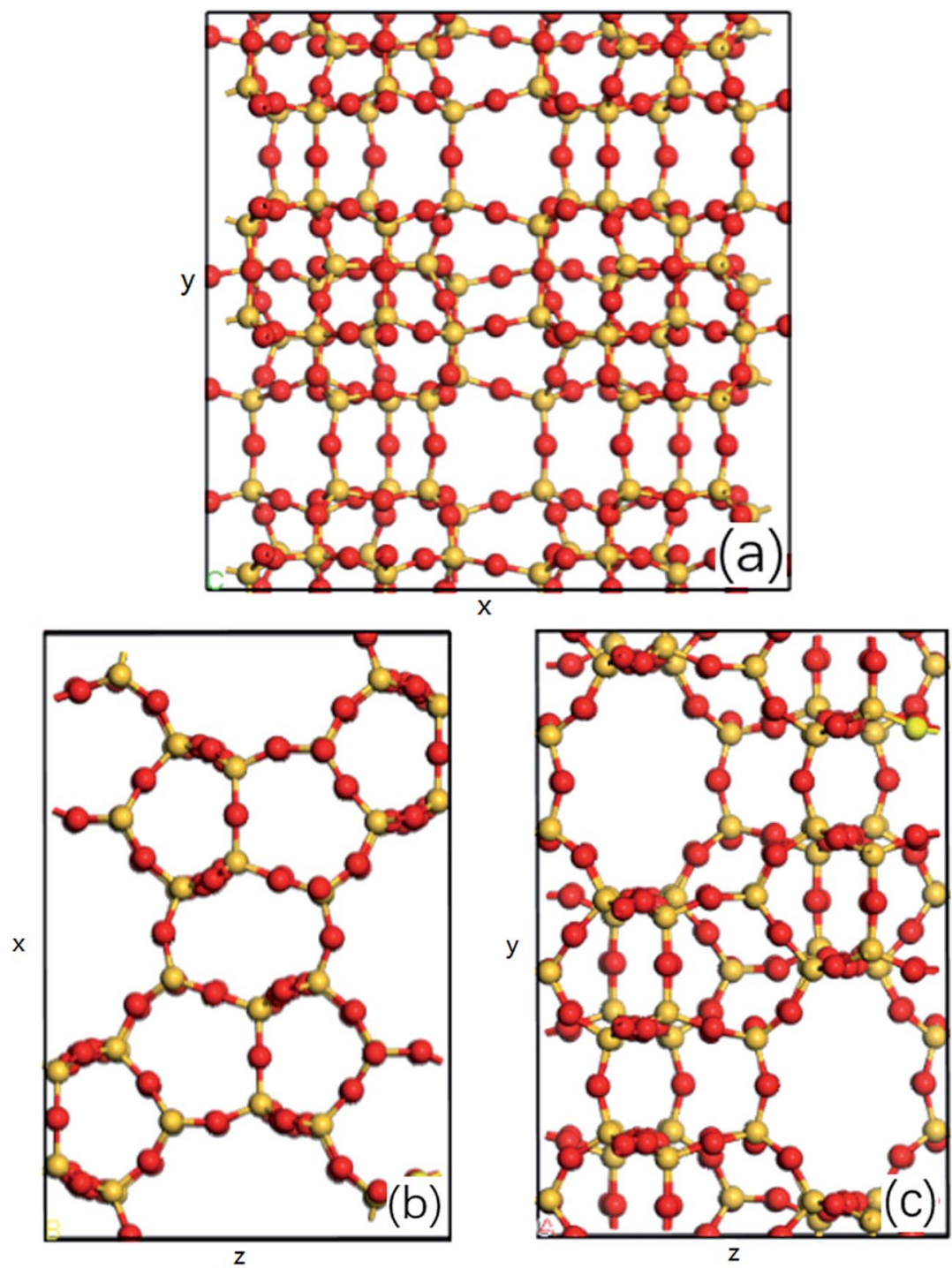

Fig. 2 (a) Main view, (b) top view and (c) left view of all-silica MFI zeolite structure, silicon atoms are represented by the yellow balls and oxygen atoms are represented by the red balls, $x, y$ and $z$ represent the edges of a unit cell in $x, y$ and $z$ direction.

For shale gas, methane is primarily in the form of adsorbed gas and free gas in nanostructures. ${ }^{4}$ The former is adsorbed on the surfaces of nanoconfinements and the latter is stored in interconnected nanopore structures. ${ }^{5-7}$ In the literature, many studies on gas adsorption and desorption in nanostructures, such as nanopores, carbon nanotubes and nanochannels, ${ }^{8-10}$ have been conducted. Rexer et al. ${ }^{6}$ experimentally studied the adsorption of methane in an alum shale sample at various temperatures and pressures corresponding to the real geological conditions. They measured and modeled the methane excess uptake and isosteric enthalpy. Their analyses are helpful for understanding the storage mechanisms of shale gas. Lithoxoos et al. ${ }^{8}$ examined the adsorption capacity of single-wall carbon nanotubes (SWCNTs) at room temperature under different pressures through experiments and computations. They obtained density profiles of different gases and found that almost all

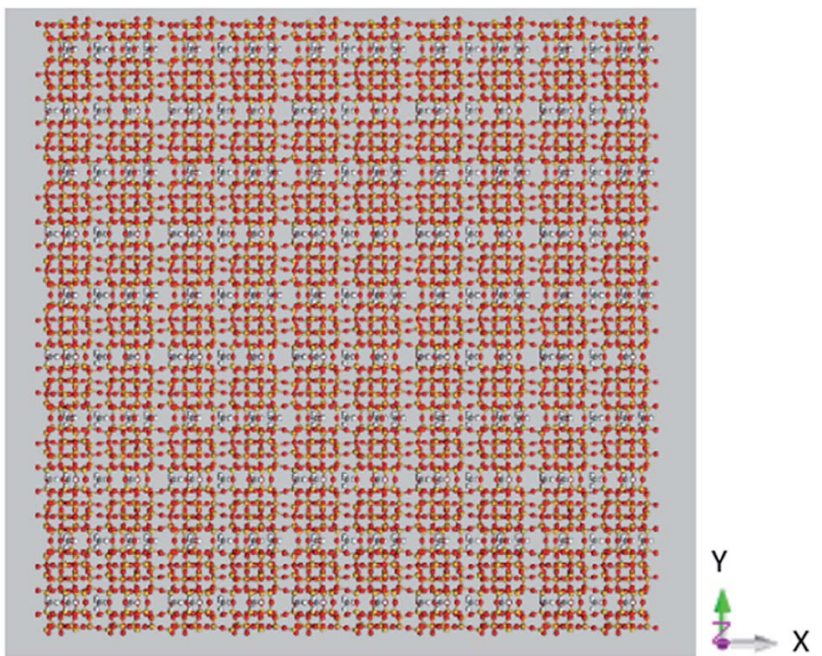

Fig. 3 A snapshot of the MD simulation system. 
Table 1 Potential parameters for the force field

\begin{tabular}{lll}
\hline Bond stretch parameters & & \\
\hline Bond type & $K_{0}\left(\mathrm{kcal} \mathrm{mol}^{-1} \AA^{-2}\right)$ & $R_{0}(\AA)$ \\
\hline $\mathrm{C}-\mathrm{H}$ & 700 & 1.09 \\
$\mathrm{Si}-\mathrm{O}$ & 700 & 1.587 \\
\hline
\end{tabular}

Bond angle bend parameters

\begin{tabular}{|c|c|c|c|}
\hline Bond type & $K_{0}\left(\mathrm{kcal} \mathrm{mol}^{-1} \AA^{-2}\right)$ & & $\theta_{0}$ \\
\hline $\mathrm{H}-\mathrm{C}-\mathrm{H}$ & 100 & & $109.471^{\circ}$ \\
\hline $\mathrm{Si}-\mathrm{O}-\mathrm{Si}$ & 100 & & $104.51^{\circ}$ \\
\hline $\mathrm{O}-\mathrm{Si}-\mathrm{O}$ & 100 & & $109.471^{\circ}$ \\
\hline \multicolumn{4}{|c|}{ Torsional parameters } \\
\hline Torsion type & $B_{0}\left(\mathrm{kcal} \mathrm{mol}{ }^{-1}\right)$ & $d_{0}$ & $n_{0}$ \\
\hline $\mathrm{O}-\mathrm{Si}-\mathrm{O}-\mathrm{Si}$ & 2 & -1 & 3 \\
\hline
\end{tabular}

Lennard-Jones parameters

\begin{tabular}{lll}
$\begin{array}{l}\text { Interaction } \\
\text { type }\end{array}$ & $\sigma(\AA)$ & $\varepsilon\left(\mathrm{kcal} \mathrm{mol}^{-1}\right)$ \\
\hline $\mathrm{H}-\mathrm{H}$ & 3.195 & 0.015 \\
$\mathrm{C}-\mathrm{C}$ & 3.898 & 0.095 \\
$\mathrm{H}-\mathrm{C}$ & 3.547 & 0.038 \\
$\mathrm{H}-\mathrm{O}$ & 3.3 & 0.038 \\
$\mathrm{H}-\mathrm{Si}$ & 3.733 & 0.069 \\
$\mathrm{C}-\mathrm{O}$ & 3.651 & 0.095 \\
$\mathrm{C}-\mathrm{Si}$ & 4.084 & 0.172 \\
\hline
\end{tabular}

Electrostatic parameters

\begin{tabular}{lc}
\hline Element type & Charge $(e)$ \\
\hline $\mathrm{C}$ & -0.572 \\
$\mathrm{H}$ & 0.143 \\
$\mathrm{O}$ & -0.55 \\
$\mathrm{Si}$ & 1.1 \\
\hline
\end{tabular}

the gas molecules are distributed near the pore walls. Zhu and Zhao ${ }^{9}$ investigated the mechanisms of methane adsorption in carbon nanopores through molecular dynamics (MD) simulations and theoretical analyses. It was found that the storage capacity of CNT is greatly enhanced compared with the bulk phase. The amount of methane molecules that can be stored in CNT at 20 bar is as much as that in the bulk phase at 220 bar. In addition, they found that there exists an optimal CNT diameter that maximizes the adsorption and developed the equation of state for adsorbed methane. $\mathrm{Wu}$ et al. ${ }^{\mathbf{1 1}}$ also explored the mechanisms of methane adsorption and displacement processes in carbon nanochannels using MD simulations and obtained similar results to those of $\mathrm{Zhu}$ and Zhao. ${ }^{9}$ They found that when the external pressure reaches 5 $\mathrm{MPa}$, the adsorption isotherms for bulk methane is about

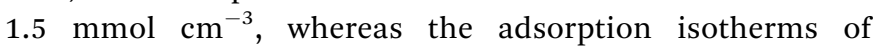
methane in silt pores is about $10 \mathrm{mmol} \mathrm{cm} \mathrm{cm}^{-3}$.

In addition to gas adsorption, gas desorption in nanopores has also been studied. Yu and Sepehrnoori ${ }^{12}$ numerically investigated the gas desorption for five different shale formations. They analyzed the factors promoting gas desorption and the negative effects of geomechanics on gas production. Shabro et al. ${ }^{13}$ implemented a new $1 \mathrm{D}$ radial model to examine the transport mechanisms of shale gases and to forecast gas production in shale-gas reservoirs. Ho et al. ${ }^{3}$ examined the pressure effects on the methane release in kerogen and found that the release of free gas is driven by pressure gradients, while the adsorbed gas is controlled by gas desorption and diffusion.

Other than pressure and pore size, temperature also plays an important role in gas desorption as it determines the mean kinetic energy of gas molecules. ${ }^{\mathbf{1 4 , 1 5}}$ Moreover, in different nanostructures, the potential distribution varies and can greatly affect the dynamics of molecules, ${ }^{\mathbf{1 6 - 1 8}}$ and will certainly affect the release percentage. Unfortunately, little work has been carried out to study the influence of temperature and pore structure on the release of methane through nanopores.

This work investigates the effects of temperature and pore structure on the release of methane in zeolite nanopores through MD simulations. The methane release percentage is obtained at different temperatures for various zeolite structures. Herein, the zeolite structure is adopted to model shale pores. Zeolite is a traditional adsorbent, which has been widely used for gas storage, such as hydrogen and methane. ${ }^{19-21}$ Molecular dynamics simulation is employed to study the methane release in the nanochannels. For all-silica

Table 2 Structural parameters of various zeolite structures

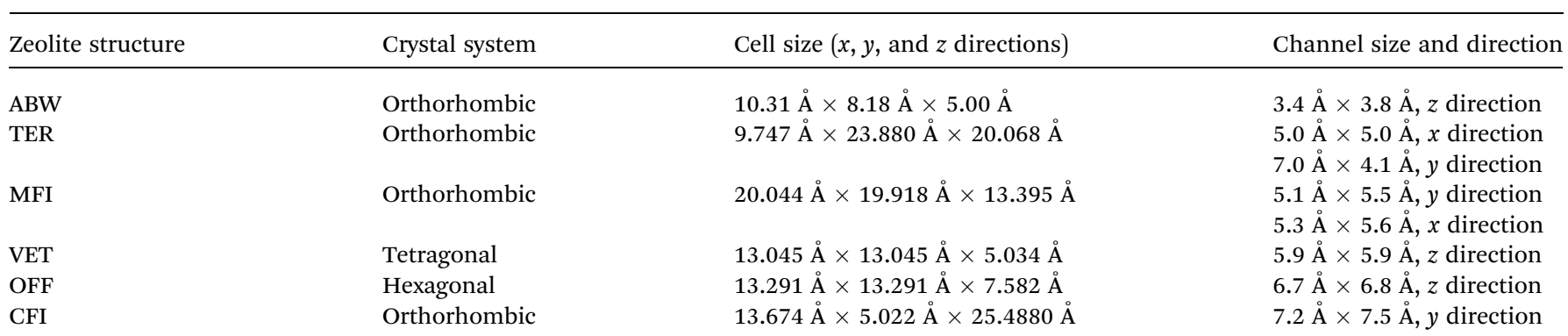



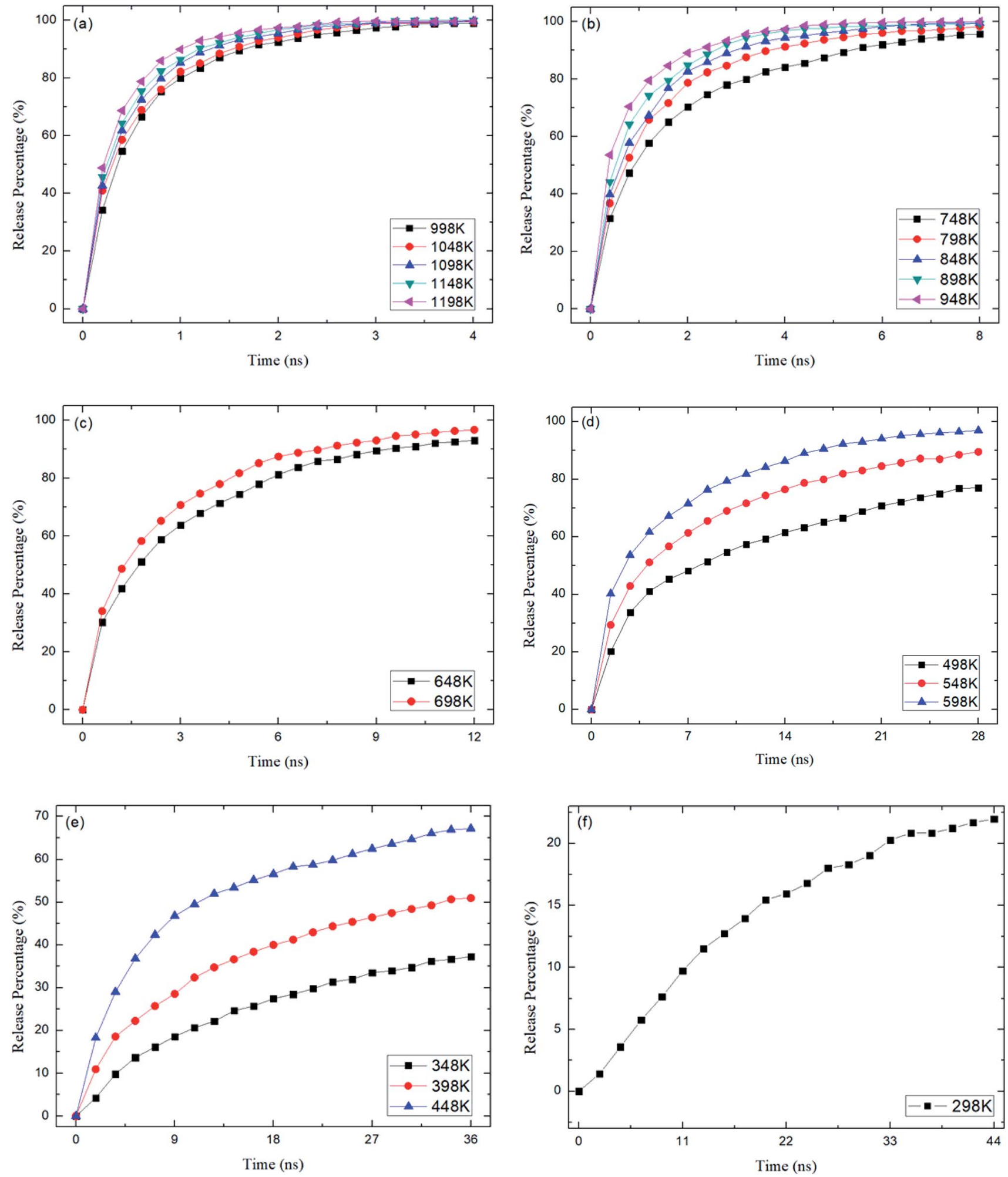

Fig. 4 Methane release percentage as a function of time at different temperatures. (a) $998 \mathrm{~K}$ to $1198 \mathrm{~K}$, (b) $748 \mathrm{~K}$ to $948 \mathrm{~K}$, (c) $648 \mathrm{~K}$ and $698 \mathrm{~K}$, (d) $498 \mathrm{~K}$ to $598 \mathrm{~K}$, (e) $348 \mathrm{~K}$ to $448 \mathrm{~K}$, (f) $298 \mathrm{~K}$.

MFI zeolite, the release percentage increases to $\sim 90 \%$ with increasing temperature before $T=598 \mathrm{~K}$, after which it rises mildly as temperature is further increased. The release of methane for different zeolite structures show that the release percentage of methane also greatly depends on the pore size. For zeolite with the average pore size of $\sim 3.5 \AA$ (ABW zeolite), 


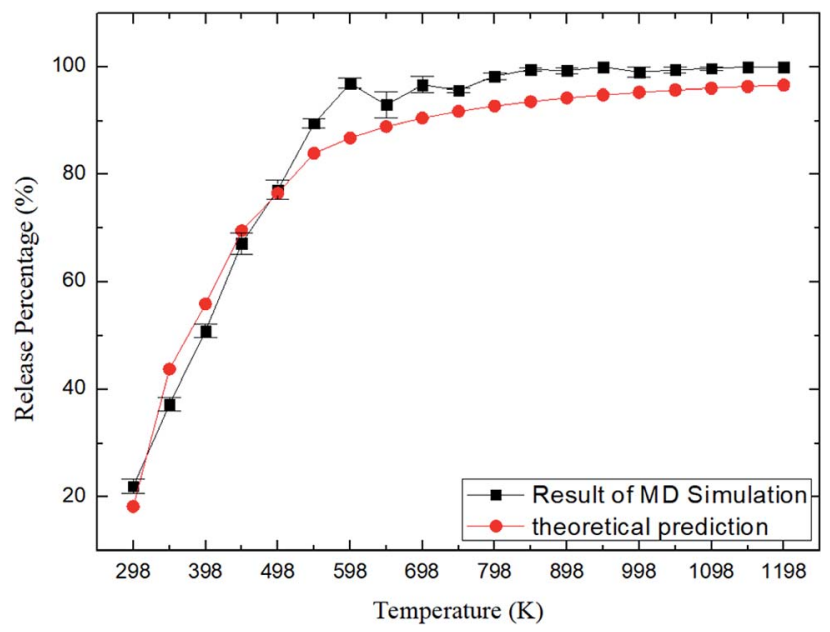

Fig. 5 Release percentage of methane as a function of temperature. Squares and circles are the results of MD simulations and theoretical predictions, respectively.

the release percentage rises from $0 \%$ to about $80 \%$ when the temperature is increased from 348 to $1148 \mathrm{~K}$. For zeolite with relatively large pore size, $7.3 \AA$ (CFI zeolite), the release percentage remains above $80 \%$ even at room temperature. The release percentage predicted using the energy barriers obtained from MD simulations shows good agreement with numerical results.

\section{Molecular dynamics simulation}

The MD simulations are performed using the commercial software, Material Studio. The nanoscale shale structures are modeled by zeolite nanochannels. The orthorhombic all-silica MFI zeolite (silicalite-1) of medium pore size is used as the major structure. The silicalite-1 structure has a threedimensional network of interconnecting channels, containing both straight channels and zigzag channels, as illustrated in Fig. 1 . The dimensions of a unit silicalite- 1 cell are $l_{x}=20.044 \AA$, $l_{y}=19.918 \AA, l_{z}=13.395 \AA$ in the $x, y$, and $z$ directions. Such a unit cell contains $96 \mathrm{SiO}_{2}$ molecules and has a pore volume of $1.83 \mathrm{~nm}^{3} .^{22}$ Fig. 2 shows different views of a silicalite-1 cell, where straight channels are in the $y$ direction and zigzag channels are in the $x z$ plane.

In simulations, a zeolite containing five unit cells in the $x$ and $y$ directions and two unit cells in the $z$ direction (totally $4800 \mathrm{SiO}_{2}$ atoms) is considered, which is $100.22,99.495$, and $26.79 \AA$ in the $x, y$, and $z$ directions, as shown in Fig. 3. The lengths of the simulation system are 50,50 , and $2.68 \mathrm{~nm}$ in the $x, y$, and $z$ directions with the zeolite at the center of the system. As periodic boundary conditions are applied in all the directions, such a configuration can eliminate the interactions between the zeolite and its images in the $x$ and $y$ directions and allows methane molecules to be released from the zeolite.
The DREIDING force field ${ }^{23}$ is used to model the zeolite, methane, and methane-zeolite interactions. The general potential for these interactions is given by

$$
\begin{aligned}
U & =\sum_{\text {bonds }} \frac{K_{0}}{2}\left(r_{\mathrm{ij}}-R_{0}\right)^{2}+\sum_{\text {angles }} \frac{K_{0}}{2}\left(\theta-\theta_{0}\right)^{2} \\
& +\sum_{\text {torsions }} \frac{B_{0}}{2}\left(1-d_{0} \cos \left(n_{0} \phi\right)\right) \\
& +\sum_{\mathrm{i}<\mathrm{j}} 4 \varepsilon_{\mathrm{ij}}\left(\left(\frac{\sigma_{\mathrm{ij}}}{r_{\mathrm{ij}}}\right)^{12}-\left(\frac{\sigma_{\mathrm{ij}}}{r_{\mathrm{ij}}}\right)^{6}\right)+\sum_{\mathrm{i}<\mathrm{j}} \frac{q_{\mathrm{i}} q_{\mathrm{j}}}{r_{\mathrm{ij}}}
\end{aligned}
$$

where $K_{0}$ is a force constant, $R_{0}$ and $\theta_{0}$ are the equilibrium bond distance and angle, $B_{0}$ is the barrier height, $d_{0}$ is the phase factor, $n_{0}$ is the periodicity, $\varepsilon$ is the binding energy and $\sigma$ is the collision diameter, $r_{\mathrm{ij}}$ is the separation between molecules/atom $\mathrm{i}$ and $\mathrm{j}, \theta$ is the bond angle, $\phi$ is the bond torsion angle, and $q$ is the charge. The values of relevant parameters for various interactions are listed in Table 1.

The cut-off distance for the potential is $10.5 \AA$ and the time step is $1 \mathrm{fs}$. The temperature of the system is maintained by the Berendsen thermostat. ${ }^{24}$ The pressure of the system is set at $20 \mathrm{MPa}$ to simulate the geological condition of practical shale gas reservoirs. ${ }^{6}$ Under this pressure, the methane density at $350 \mathrm{~K}$ is determined as $119.41 \mathrm{~kg} \mathrm{~m}^{-3},{ }^{25}$ which is used to disperse methane molecules in the system. The loading of methane is calculated based on this density from the equation of state ${ }^{25}$ according to the pore volume (1.83 $\mathrm{nm}^{3}$ per unit cell). Initially, both the free space outside and the pores inside the zeolite are filled with methane molecules according to this density. The molecules outside the zeolite are used to help make the conditions inside the zeolite similar to the real geological conditions of shale gases. The system is relaxed for $200 \mathrm{ps}$ in the $(N, V, T)$ ensemble. Then the methane molecules outside the zeolite are removed and the new system with methane inside the zeolite is performed in the $(N, V, E)$ ensemble for calculating the release percentage, as shown in Fig. 3. Depending on the temperature of the system, the simulation time ranges from 4 to 45 ns, which is sufficiently long for the system to reach the steady state.

In addition to all-silica MFI zeolite, five other zeolite structures, i.e. ABW, VET, TER, CFI and OFF zeolites, are also considered, which have different pore sizes. The structural parameters of these zeolites are given in Table $2 .^{26}$

\section{Results and discussion}

The number of methane molecules released from the zeolites is obtained in the simulations and the release percentage is calculated, which is defined as

$$
P_{0}=\frac{N_{0}}{N} \times 100 \%
$$



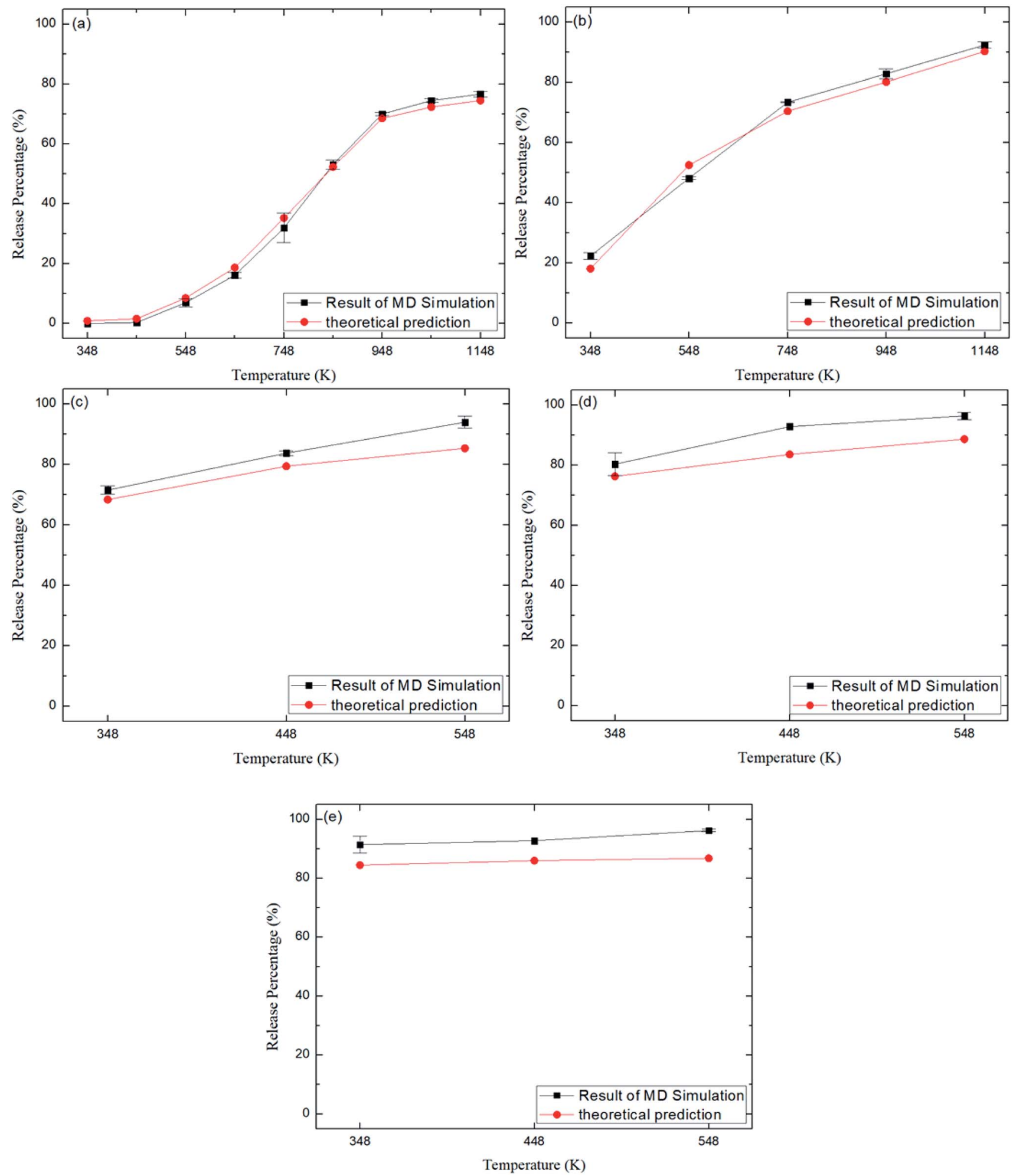

Fig. 6 Release percentage of methane in different zeolite structures in Table 2. (a) ABW. (b) TER. (c) VET. (d) OFF. (e) CFI. Squares and circles are the results of MD simulations and theoretical predictions, respectively.

where $N_{0}$ is the number of methane molecules released from the zeolite and $N$ is the total number of methane molecules initially confined in the zeolite. It is noted that the release process is accompanied by the reabsorption of methane on zeolite pore surface and $N_{0}$ is the net molecule number due to the two processes. Fig. 4 shows the release percentage as 

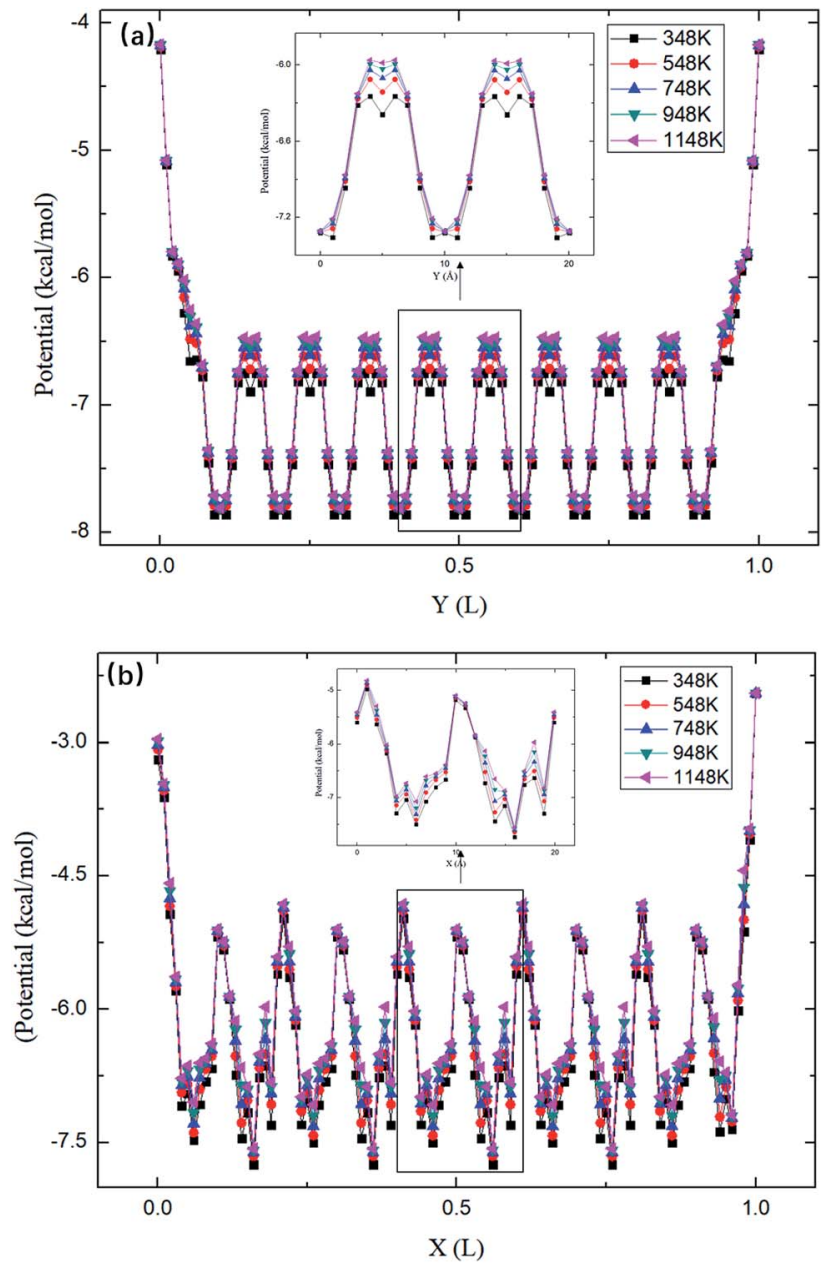

Fig. 7 The average potential profile of silicalite-1 structure. (a) Straight channel and (b) zigzag channel, where $L$ is the length of the corresponding channel.

a function of time at different temperatures for all-silica MFI zeolite. It is seen that the time needed to release methane molecules depends on the temperature. The release process takes about 4 to $45 \mathrm{~ns}$ when the temperature $T$ is varied from 298 to 1198 K. Fig. 5 depicts the final release percentage $P_{0}$ as a function of temperature. It is clear that $P_{0}$ increases with increasing $T$ for $T<598 \mathrm{~K}$. Beyond $598 \mathrm{~K}, P_{0}$ is roughly a constant, above $90 \%$, indicating that most methane molecules are released from the zeolite. Further increase in temperature does not have significant effect on the methane release.

Fig. 6 depicts the final release percentages $P_{0}$ for other zeolite structures at different temperatures. It is seen that at a given temperature, $P_{0}$ generally increases with increasing pore size. For instance, at $348 \mathrm{~K}$, the release percentage $P_{0} \approx$ $90 \%$ for CFI zeolite with average pore size of $7.3 \AA$ (Table 2), as shown in Fig. 6e, while the release percentage is $0 \%$ for $\mathrm{ABW}$ zeolite due to the small pore size ( 3.5 $)$ (Fig. 6a).

The dynamics of molecules in a pore mainly depends on the kinetic energy $E$ of the molecules and the potential distribution inside the pore..$^{27,28}$ The former is determined by the temperature and the latter is governed by the structure of the pore and the molecular interactions. To be released from the zeolite, a methane molecule should have a sufficiently high kinetic energy to overcome potential energy barriers inside the pores. According to the Maxwell-Boltzmann velocity distribution, the kinetic energy distribution of the methane molecules follows

$$
f(E)=2 \sqrt{\frac{E}{\pi}}\left(\frac{1}{k_{\mathrm{B}} T}\right)^{3 / 2} \mathrm{e}^{\left(\frac{-E}{k_{\mathrm{B}} T}\right)}
$$

where $k_{\mathrm{B}}$ is the Boltzmann constant. If the maximum potential energy barrier in the zeolite nanochannels is denoted as $\Delta G$, the release percentage can be theoretically predicted by

$$
P^{*}=1-\int_{0}^{\Delta G} 2 \sqrt{\frac{E}{\pi}}\left(\frac{1}{k_{\mathrm{B}} T}\right)^{3 / 2} \mathrm{e}^{\left(\frac{-E}{k_{\mathrm{B}} T}\right)} \mathrm{d} E
$$

The maximum potential energy barrier $\Delta G$ depends on the molecular interactions and the pore size. Fig. 7 shows the average potential energy along the pore axis, $U_{\text {avg }}(z)$, calculated through

$$
U_{\text {avg }}(z)=\frac{\int_{x} \int_{y} U(x, y, z) \mathrm{e}^{-\beta U(x, y, z)} \mathrm{d} x \mathrm{~d} y}{\int_{x} \int_{y} \mathrm{e}^{-\beta U(x, y, z)} \mathrm{d} x \mathrm{~d} y},
$$

where $\beta=1 / k_{\mathrm{B}} T$. It is seen that the energy barrier of zigzag channels is much higher than that in straight channels. Thus, for a molecule initially lying in a zigzag channel, it is very probable that it will migrate to the intersection of a straight channel and the zigzag channel and then is released from the zeolite through the straight channel, as illustrated in Fig. 7.

As shown in Fig. 7, the energy barriers, $\Delta G=3.651,3.527$, $3.434,3.208,3.102 \mathrm{kcal} \mathrm{mol}^{-1}$ at $348,548,748,948$, and 1148 $\mathrm{K}$, respectively. These energy barriers can be used to obtain the theoretical release percentages using eqn (4). The release percentage predicted by eqn (4) is also shown in Fig. 5. It is see that the theoretical predictions agree well with the MD results. Eqn (4) slightly underestimates the release percentage at high temperatures because it does not consider the fluctuation in kinetic energy and potential energy barrier.

Fig. 8 depicts the energy barriers in the pores of other zeolite structures. It is seen that $\Delta G$ in $\mathrm{ABW}$ pores is the largest and that for CFI is the smallest, which are consistent with the release percentages shown in Fig. 6. Fig. 6 also shows the release percentages predicted by eqn (4). It is seen that eqn (4) works well for all the zeolite structures. 

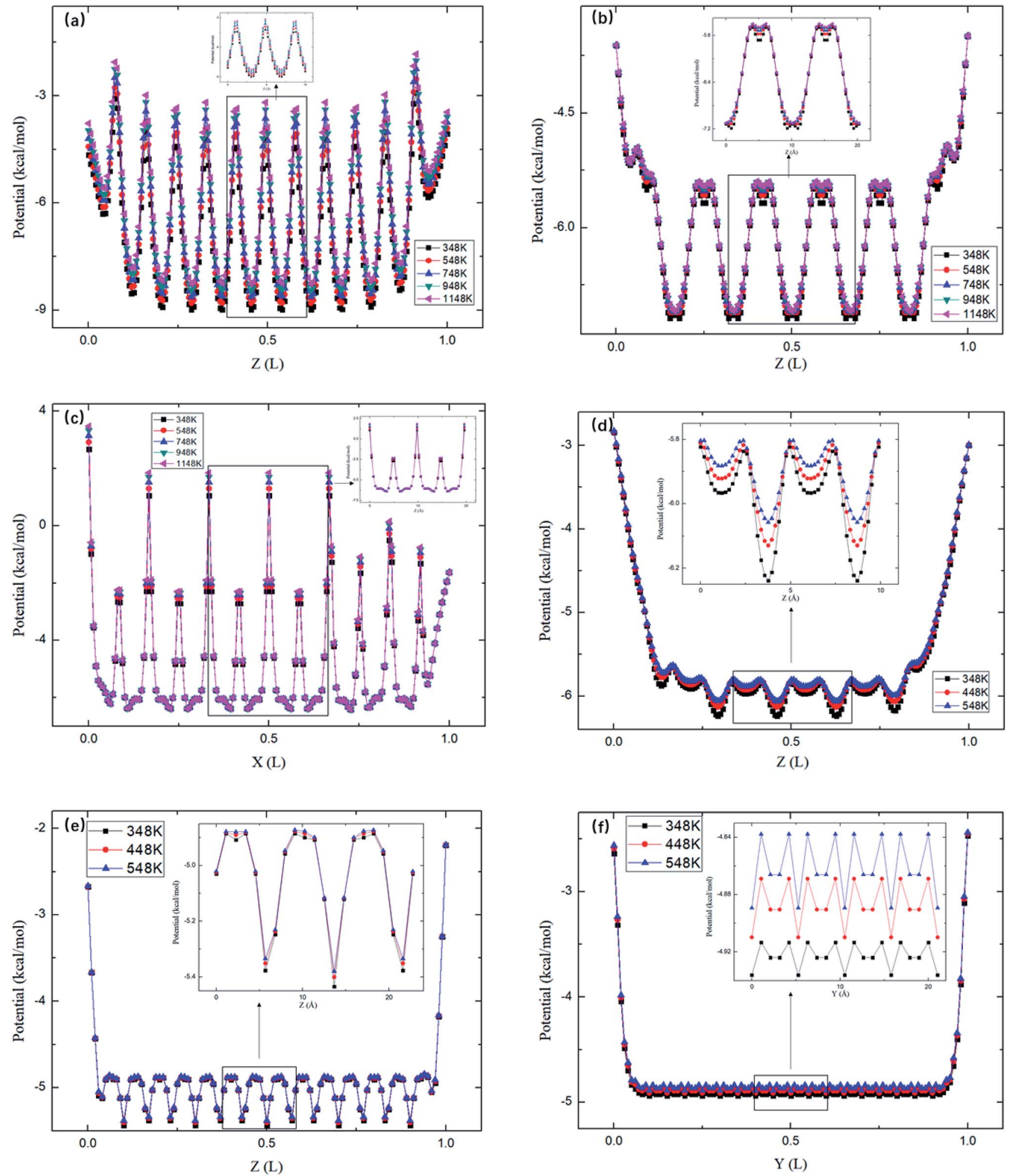

Fig. 8 The average potential profile of zeolite structures in Table 2. (a) ABW. (b) Straight channels of TER. (c) Zigzag channels of TER. (d) VET. (e) OFF. (f). CFI, where $L$ is the length of the corresponding channel.

\section{Conclusions}

The effects of the temperature as well as pore size on the release of methane in zeolite nanochannels have been examined by MD simulations. The methane release percentage in MFI zeolite nanochannels increases with increasing temperature when $T<598 \mathrm{~K}$. Above $598 \mathrm{~K}$, most of the methane molecules are released from the zeolite and the 
release percentage remains roughly constant at a high value. Simulations for other zeolite structures show that methane molecules are apt to escape from nanoconfinements with large pore sizes, which have relatively low energy barriers. Theoretical predictions of the release percentages using the energy barriers obtained through MD simulations agree well with numerical results.

\section{Conflicts of interest}

There are no conflicts to declare.

\section{Acknowledgements}

This work was supported by the Research Grants Council of the Hong Kong Special Administrative Region under Grant No. 16210615, the National Natural Science Foundation of China (No. 51888103), and the Foundation for Innovative Research Groups of the National Natural Science Foundation of China (No. 51721004).

\section{References}

1 J. Chen, F. Wang, H. Liu and H. Wu, Sci. China: Phys., Mech. Astron., 2016, 60, 014611.

2 J. D. Hughes, Nature, 2013, 494, 307-308.

3 T. A. Ho, L. J. Criscenti and Y. Wang, Sci. Rep., 2016, 6, 28053.

4 R. G. Loucks, R. M. Reed, S. C. Ruppel and D. M. Jarvie, J. Sediment. Res., 2009, 79, 848-861.

5 S. L. Montgomery, D. M. Jarvie, K. A. Bowker and R. M. Pollastro, AAPG Bull., 2005, 89, 155-175.

6 T. F. T. Rexer, M. J. Benham, A. C. Aplin and K. M. Thomas, Energy Fuels, 2013, 27, 3099-3109.

7 W. Zhou, Z. Zhang, H. Wang, Y. Yan and X. Liu, RSC Adv., 2018, 8, 33939-33946.

8 G. P. Lithoxoos, A. Labropoulos, L. D. Peristeras, N. Kanellopoulos, J. Samios and I. G. Economou, J. Supercrit. Fluids, 2010, 55, 510-523.
9 X. Zhu and Y. P. Zhao, J. Phys. Chem. C, 2014, 118, 1773717744.

10 N. Zucchetto and D. Brühwiler, RSC Adv., 2015, 5, 7463874644.

$11 \mathrm{H}$. Wu, J. Chen and H. Liu, J. Phys. Chem. C, 2015, 119, 13652-13657.

12 W. Yu and K. Sepehrnoori, Fuel, 2014, 116, 455-464.

13 V. Shabro, C. Torres-Verdin and K. Sepehrnoori, SPE Annual Technical Conference and Exhibition, Society of Petroleum Engineers, San Antonio, Texas, USA, 2012.

14 Z. Li and H. Wang, Phys. Rev. Lett., 2005, 95, 014502.

15 Z. Li and H. Wang, Phys. Rev. E: Stat., Nonlinear, Soft Matter Phys., 2003, 68, 061206.

16 L. Li, J. Mo and Z. Li, Phys. Rev. Lett., 2015, 115, 134503.

17 J. Mo, L. Li, J. Zhou, D. Xu, B. Huang and Z. Li, Phys. Rev. E: Stat., Nonlinear, Soft Matter Phys., 2015, 91, 033022.

18 J. Mo, C. Li, L. Li, J. Wang and Z. Li, Phys. Fluids, 2016, 28, 082005.

19 D. P. Broom and K. M. Thomas, MRS Bull., 2013, 05, 412-421.

20 K. S. Smirnov and B. van de Graaf, J. Chem. Soc., Faraday Trans., 1996, 92, 2475-2480.

21 J. Dong, X. Wang, H. Xu, Q. Zhao and J. Li, Int. J. Hydrogen Energy, 2007, 18, 4998-5004.

22 C. Tedesco, L. Erra, M. Brunelli, V. Cipolletti, C. Gaeta, A. N. Fitch, J. L. Atwood and P. Neri, Chem.-Eur. J., 2010, 16, 2371-2374.

23 S. L. Mayo, B. D. Olafson and W. A. Goddard, J. Phys. Chem., 1990, 94, 8897-8909.

24 H. J. C. Berendsen, J. P. M. Postma, W. F. V. Gunsteren, A. Dinola and J. R. Haak, J. Chem. Phys., 1984, 81, 3684-3690.

25 U. Setzmann and W. Wagner, J. Phys. Chem. Ref. Data, 1991, 20, 1061-1155.

26 C. Baerlocher, W. M. Meier and D. H. Olson, Atlas of Zeolite Framework Types, Elsevier, 2007, pp. 174-175.

27 R. L. June, A. T. Bell and D. N. Theodorou, J. Phys. Chem., 1990, 94, 8232-8240.

28 Q. Yang and C. Zhong, J. Phys. Chem. B, 2006, 110, 1777617783. 